\title{
Colecistectomia laparoscópica en colelitiasis y situs inversus totalis. Reporte de caso con evolución favorable
}

\author{
Rafael Coello Cuntó ${ }^{1,2}$; Martha Silva Chipe ${ }^{1}$; Alex Vivas Lara ${ }^{1,2}$; Donny Peñafiel Pazmiño ${ }^{1}$ Christian Ortiz Muñoz ${ }^{3}$, \\ Rafael Coello Salguero ${ }^{4}$
}

\section{RESUMEN}

El situs inversus totalis(SIT) es una afección infrecuente no invalidante; su presencia no predispone a la colelitiasis. La colecistectomía laparoscópica (CL) puede realizarse sin mayor riesgo pero con alguna dificultad; la conversión es posible cuando se encuentra una variación anatómica acompañante; se presenta un caso conocido de SIT portador de colelitiasis intervenido quirúrgicamente con evolución favorable.

Palabras clave: Colecistectomía, Laparoscopia, situs inversus totalis, Trígono cistohepático.

\section{Laparoscopic cholecystectomy in cholelithiasis and situs inversus totalis. Case report with favourable evolution}

\begin{abstract}
Situs inversus totalis (SIT) is a rare, non-disabling condition; its presence does not predispose to cholelithiasis. Laparoscopic cholecystectomy (LC) can be performed without increased risk but with some difficulty; conversion is possible when an accompanying anatomic variation is found; We present a known case of SIT with cholelithiasis who underwent surgery with favorable evolution.
\end{abstract}

Key words: Cholecystectomy, Laparoscopy, situs inversus totalis, Cystohepatic trigone.

\section{Introducción}

Descrita por primera vez en 1600 por Fabricus (1) el situs inversus totalis (SIT) es infrecuente y se considera una variación debida a desorden autosómico recesivo (2) con una incidencia de 1:10000 a 1:20000 nacidos vivos(3) pudiendo acompañarse de otras anomalías como displasia renal, atresia biliar, alteraciones cardiacas o fibrosis pancreática(4). Comparados con la prevalencia del resto de la población, se conocen pocos casos de SIT con colelitiasis (5) sin haberse demostrado que la SIT aumente la presencia de colelitiasis $(6,7)$.

La CL es el tratamiento de elección para la colelitiasis sintomática incluido el paciente portador de SIT (4, 8) pudiendo ser dificultoso la CL en estos casos por la inversión anatómica de las vísceras. Las dificultades y eventuales complicaciones de una colecistectomía clásica se podrían considerar de mayor frecuencia si la vesícula biliar se encuentra en posición opuesta; sin embargo, se sabe que la CL trascurre sin mayores riesgos. Se han reportado casos de colelitiasis en paciente con SIT (5) que provocan una reorientación en la estrategia quirúrgica; por lo inusual de la presentación congénita, se reporta un caso de adulto portador de SIT con colelitiasis intervenido

1. Especialista en Cirugía General . Hospital de especialidades Teodoro Maldonado Carbo IESS.

2. Carrera de Medicina. Universidad de Guayaquil.

3. Medico Residente Hospital IESS- Los Ceibos . Guayaquil- Ecuador

4. Especialista en Gineco-Obstetricia. Hospital de Especialidades Teodoro Maldonado Carbo IESS

Citar como : Coello Cuntó R., Silva Chipe M., Vivas Lara A., Donny Peñafiel Pazmiño D., Ortiz Muñoz. Ch, Coello Salguero R., Colecistectomia laparoscópica en colelitiasis y situs inversus totalis. Reporte de caso con evolución favorable. Rev Peruana de Morfologia. 2021; 2(1):38-41, doi:https//doi.org/1051343/revperuanamorfologia.v2i1.271

Recibido: 2020-06-11; Aceptado: 2020-12-29

Autor corresponsal: Dr. Rafael Coello Cuntó, Phd., Mg.Esp.; Email: msdracocu@hotmail.com 
quirúrgicamente, cuyo diagnóstico se comprobó por medio de ecosonografía y tomografía simple.

\section{Descripción del caso}

El paciente masculino de 32 años casado sin hijos conocido portador de SIT(Q89.3-ICD10; CI10-OMS) (LA82CIE11, ICD11) sin antecedente patologico de interés ni evidencias familiares de alteraciones congénitas hasta el primer nivel de consanguinidad en relación historia clinica del portador; examen de laboratorio reportó buen funcionamiento hepático, renal y hemostático; la ecosonografía mostró litiasis vesicular y ausencia de dilatación de vías biliares, por medio de Radiografica de tórax se presentó la silueta cardiaca hacia la derecha sin evidencia de bronquiectasia (Fig.1), detalles del diagnóstico se ratificaron por medio de tomografía simple (Figs.2 y 3) que mostraba ubicación de vísceras torácica y abdominales en posición inversa: hígado en hipocondrio izquierdo y bazo en hipocondrio derecho, burbuja aérea subdiafragmática derecha que corresponde a colon. El paciente había presentado leve dolor en epigastrio e hipocondrio izquierdo y molestias después de cada comida, asintomático respiratorio, afebril y anictérico. Los antecedentes de eventual situs inversus surgieron durante la historia clínica; la valoración cardiológica y el electrocardiograma revelaron dextrocardia.

Por presentar colelitiasis sintomática fue intervenido quirúrgicamente en el Servicio de Cirugía General del Hospital de Especialidades del IESS Teodoro Maldonado Carbo de Guayaquil-Ecuador con buena evolución posoperatoria.

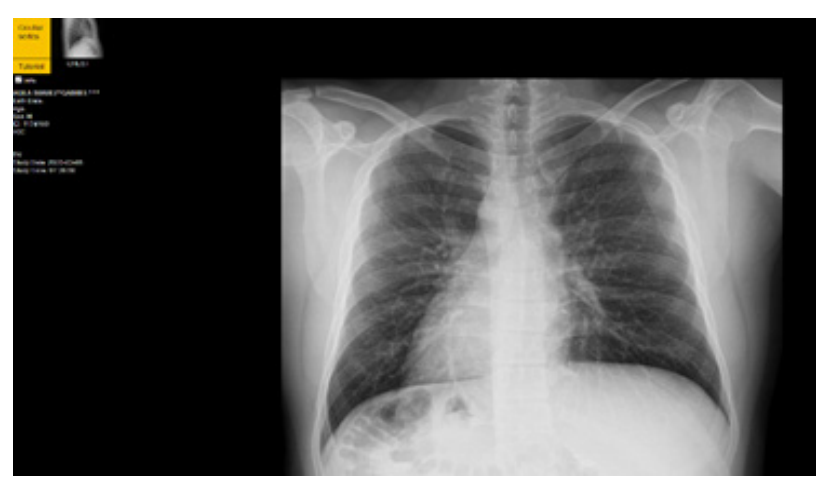

Fig. 1. radiografia de torax que muestra silueta cardiaca hacia la derecha

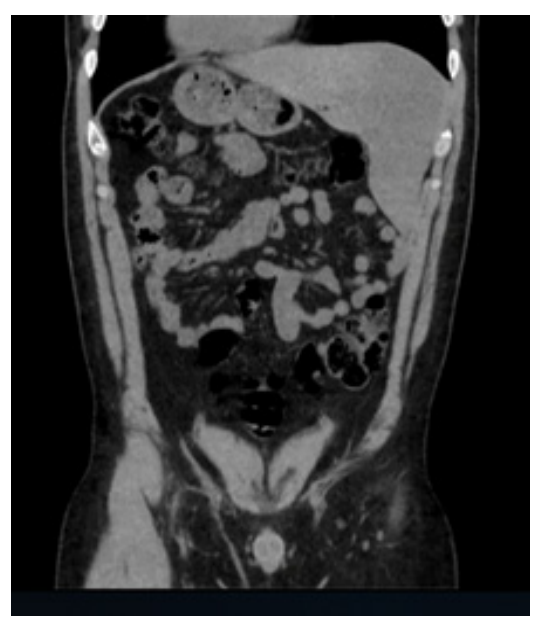

Fig 2. tomografia axial de abdomen que muestra higado a la izquierdad.

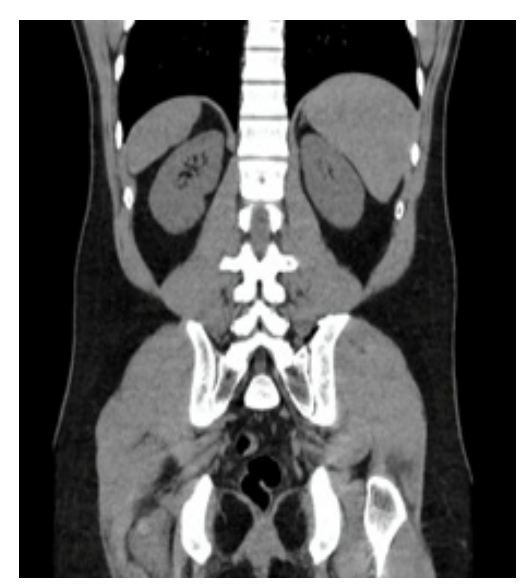

Fig 3. tomografia axial de abdomen que muestra higado a la izquierdad. y bazo a la derecha

\section{Técnica desarrollada}

Previo consentimiento informado, la CL programada se realizó bajo anestesia general utilizando 4 trócares: de $10 \mathrm{~mm}$, en posición umbilical y otro subxifoideo; de 5 $\mathrm{mm}$ en flanco izquierdo y otro en línea medio clavicular izquierda instalados bajo visión laparoscópica. El equipo quirúrgico y todos los dispositivos laparoscópicos se ubicaron en situación inversa a la distribución establecida en una CL estándar: el monitor se ubicó en relación al hombro izquierdo del paciente(Fig.4). Se aplicó neumoperitoneo en forma abierta por puerto umbilical a una presión de $12 \mathrm{mmHg}$ sin dificultad. Durante la exploración abdominal inicial se comprobó la inversión visceral; la ubicación del equipo quirúrgico fue la siguiente: el cirujano y el primer asistente con la cámara se ubicaron a la derecha y el segundo asistente a la izquierda. Una alternativa ergonométrica del cirujano fue utilizar su 
mano derecha para la disección por el puerto subxifoideo y con la mano izquierda tomar el fondo vesicular; de modo que el segundo asistente podía traccionar el bacinete hacia la derecha o realizarlo el primer asistente (9) pero también puede ser utilizada esta otra estrategia: traccionar a través del puerto epigástrico y operar a través del puerto subcostal(10); después de la disección se identificó el TCH ubicándose la arteria cística medial al ducto cístico; estos elementos se seccionan entre clics; luego se realizó disección de la vesícula biliar en forma retrógada que consumió un tiempo mayor que lo habitual (9) y se retiró la vesícula biliar por puerto umbilical sin complicaciones; el tiempo quirúrgico fue aproximadamente $60 \mathrm{~min}$. El paciente fue dado de alta hospitalaria en 24 horas y dos posteriores controles revelaron buena evolución, el reporte de la patología fue colelitiasis.

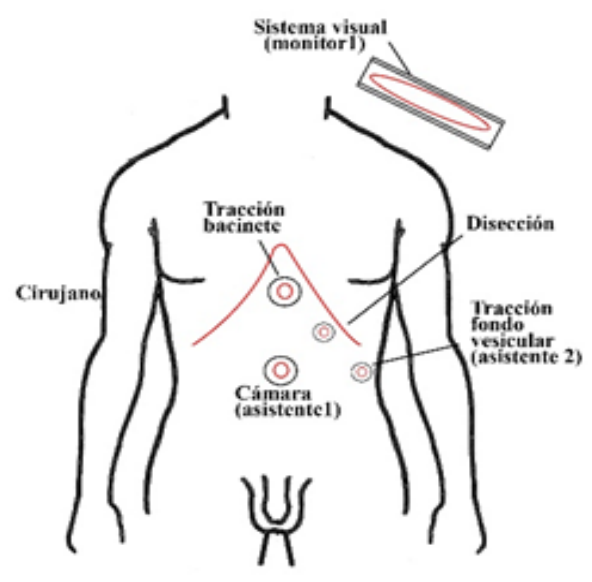

Fig 4. Distribución de los puertos y equipo quirúrgicos.

\section{DISCUSIÓN}

El paciente con SIT y colelitiasis puede presentar dolor abdominal alto, lo que puede suceder porque el sistema nervioso autónomo no participa de la trasposición visceral $(6,11)$ hecho que obliga a tener suspicacia por el diagnostico en ausencia de imágenes. La etiología del SIT no está bien definido; se presenta en una incidencia de sexo por igual 1:1 y no tiene predilección por raza (12) y en ocasiones fortuitas se detecta el SIT en presencia de otras situaciones clínicas (13) pudiendo ser acompañada de alguna variación vascular (14). La reversión visceral puede ser torácica, abdominal o ambos, producto de la rotación de $270^{\circ}$ en el sentido del reloj (8). La primera CL reportada en SIT fue en 1991 por Campos y Sipes $(1,15)$. La infrecuencia de la presentación se destaca por haberse reportado desde 1991 hasta el 2001, 32 casos que fueron tratados con técnica abierta (16) y aproximadamente 20 casos por CL hasta el 2009 (17) 36 por vía laparoscópica hasta el 2010 ( Vijay Bangaokar) y hasta el 2011 solo 50 casos por vía cerrada (18). Pitiakoudis et al., (2005), publicaron una revisión de 25 casos. Bediu et al. (2006), reportaron 13 casos de SIT por CL y en 2016 suben a 67 (1). Los reportes indican que la CL es dificultosa en pacientes con SIT pero este cuadro no contraindica la CL $(2,4)$. Si el paciente ya tiene diagnóstico de SIT desde la infancia es más ventajoso comparando con aquellos casos que clínicamente se debe sospechar por lo confuso de sus síntomas (17). A la fecha no se conoce que se haya convertido un caso a cirugía abierta atribuible a la mayores precauciones adoptadas en SI (19).

Si bien no existe un protocolo definido sobre la CL en estos casos, la cirugía es un desafío que debe ser atendido por equipo experto; la utilización de una colangiografía clásica o fluorescente transoperatoria puede ser de ayuda para disminuir los riesgos de lesiones biliares, especialmente para un equipo con poca experiencia en cirugía biliar (20); los desafíos del plan quirúrgico se remiten a las maniobras sin cruzar los miembros superiores, utilizando de preferencia el puerto epigástrico para la disección y el puerto en la línea medio axilar izquierda para la retracción del infundíbulo ubicándose el cirujano a la derecha del paciente (21); la disposición del equipo y los puertos son considerados aspectos secundarios, debiendo siempre tomarse las medidas óptimas, mas favorables y aplicando las mejores destrezas del cirujano para la disección del TCH (4). No se conocen casos intervenidos quirúrgicamente publicados en el Hospital del IESS Teodoro Maldonado Carbo de Guayaquil-Ecuador.

\section{CONCLUSIONES}

Por la condición inusual del caso, el acto operatorio implica un desafío abordar la región hepatobiliar con visión invertida a la estándar. Los desafíos técnicos incluyen: ubicación de los puertos en espejo y la disección del TCH que garanticen una buena disección; sin embargo se estima que el procedimiento es factible, seguro, con resultados similares al procedimiento estándar. Con un buen plan quirúrgico la CL en SIT siempre requiere de mayor habilidad, maniobras adoptadas durante el desarrollo el procedimiento, equipo bien entrenado y de preferencia un cirujano ambidiestro.

\section{Fuente de Financiación}

Autofinanciado

\section{Conflicto de Interés}

El autor manifiesta que no existe ningún tipo de conflicto 


\section{REFERENCIAS}

1. Alsabek MB, Arafat S, Aldirani A. A case report of laparoscopic cholecystectomy in situs inversus totalis: Technique and anatomical variation. International Journal of Surgery Case Reports. 2016;28:124-6.

2. Arya SV, Das A, Singh S, Kalwaniya DS, Sharma A, Thukral BB. Technical difficulties and its remedies in laparoscopic cholecystectomy in situs inversus totalis: A rare case report. International Journal of Surgery Case Reports. 2013;4(8):727-30.

3. Al-Jumaily M, Hoche F. Laparoscopic cholecystectomy in situs inversus totalis: is it safe? J Laparoendosc Adv Surg Tech A. 2001;11(4):229-31.

4. Ali MS, Attash SM. Laparoscopic cholecystectomy in a patient with situs inversus totalis: case report with review of literature. BMJ Case Reports. 2013;2013:bcr2013201231.

5. Elbeshry TM, Ghnnam WM. Retrograde (fundus first) Laparoscopic Cholecystectomy in Situs Inversus Totalis. Sultan Qaboos Univ Med J. 2012;12(1):113-5.

6. Hall T, Barandiaran J, Perry E. Laparoscopic cholecystectomy in situs inversus totalis: is it safe? The Annals of The Royal College of Surgeons of England. 2010;92(5):e30-e2.

7. McKay D, Blake G. Laparoscopic cholecystectomy in situs inversus totalis: a case report. BMC Surgery. 2005;5(1):5.

8. Patle NM, Tantia O, Sasmal PK, Khanna S, Sen B. Laparoscopic Cholecystectomy In Situs Inversus - Our Experience of 6 Cases. Indian Journal of Surgery. 2010;72(5):391-4.

9. $\mathrm{Mn} \mathrm{R}, \mathrm{S} \mathrm{MS}, \mathrm{Bb} \mathrm{SK}$. Laparoscopic cholecystectomy in situs inversus totalis. J Clin Diagn Res. 2014;8(7):ND03-ND5.

10. Uludag M, Yetkin G, Kartal A. Singleincision laparoscopic cholecystectomy in situs inversus totalis. JSLS. 2011;15(2):23943.

11. Takei HT, Maxwell JG, Clancy TV, Tinsley EA. Laparoscopic cholecystectomy in situs inversus totalis. J Laparoendosc Surg. 1992;2(4):171-6

12. Tsoucalas G, Thomaidis V, Fiska A. Situs inversus Totalis: Always recall the uncommon. Clinical Case Reports. 2019;7(12):2575-6.

13. Edzie EKM, Dzefi-Tettey $\mathrm{K}$, Cudjoe O, Gorleku PN, Adu P. Incidental Finding of Dextrocardia with Situs Inversus in a 59-Year-Old Man. Case Reports in Radiology. 2019;2019:7107293.

14. Kulesza RJ, Jr., Kalmey JK, Dudas B, Buck WR. Vascular anomalies in a case of situs inversus. Folia Morphol (Warsz). 2007;66(1):69-73.
15. Campos L, Sipes E. Laparoscopic cholecystectomy in a 39-year-old female with situs inversus. J Laparoendosc Surg. 1991;1(2):123-5; discussion 6.

16. Yaghan RJ, Gharaibeh KI, Hammori $\mathrm{S}$. Feasibility of laparoscopic cholecystectomy in situs inversus. J Laparoendosc Adv Surg Tech A. 2001;11(4):233-7.

17. Eisenberg D. Cholecystectomy in situs inversus totalis: a laparoscopic approach. Int Med Case Rep J. 2009;2:27-9.

18. Salama IA, Abdullah MH, Houseni M. Laparoscopic cholecystectomy in situs inversus totalis: Feasibility and review of literature. International Journal of Surgery Case Reports. 2013;4(8):711-5.

19. Machado NO, Chopra P. Laparoscopic cholecystectomy in a patient with situs inversus totalis: feasibility and technical difficulties. JSLS. 2006;10(3):386-91.

20. Rungsakulkij N, Tangtawee P. Fluorescence cholangiography during laparoscopic cholecystectomy in a patient with situs inversus totalis: a case report and literature review. BMC Surgery. 2017;17(1):43.

21. Alam A, Santra A. Laparoscopic cholecystectomy in a case of situs inversus totalis: a review of technical challenges and adaptations. Ann Hepatobiliary Pancreat Surg. 2017;21(2):84-7. 